\title{
Ganglioside GD2 expression is maintained upon recurrence in patients with osteosarcoma
}

\author{
Vincent I Poon ${ }^{1}$, Michael Roth${ }^{1}$, Sajida Piperdi ${ }^{1}$, David Geller ${ }^{2}$, Jonathan Gill ${ }^{1}$, Erin R Rudzinski ${ }^{3}$, Douglas S Hawkins ${ }^{4}$ \\ and Richard Gorlick ${ }^{1,5^{*}}$
}

\begin{abstract}
Background: Osteosarcoma is the most common primary malignant bone tumor in children and young adults. Ganglioside GD2 has been previously found on the cell surface in various tumor types, including osteosarcomas.

Findings: In this study, forty-nine additional osteosarcoma samples from 14 individual patients were assessed for GD2 expression via immunohistochemistry, of which 47 samples were found to express GD2. In matched samples from patients, GD2 expression seen at initial biopsy was found to persist in 100\% of tissues taken at recurrence.
\end{abstract}

Conclusions: GD2 expression was found to persist upon recurrence. These results suggest a phase 2 trial in children with recurrent osteosarcoma should provide an appropriate read out on the efficacy of anti-GD2 antibody.

Keywords: Osteosarcoma, Ganglioside GD2, Immunotherapy, Antibody

\section{Findings}

Osteosarcoma is the most common primary malignancy of the bone in children and young adults. Survival outcomes for children with osteosarcoma have remained stagnant for the past three decades. Novel therapeutic approaches are needed to improve outcomes for these patients. Studies have demonstrated that targeting the ganglioside GD2 in patients with high risk neuroblastoma improves survival [1]. Our group recently reported that GD2 is expressed in osteosarcoma, and the data suggested GD2 expression may be increased in relapsed specimens. However, the previous study lacked matched samples to make definitive conclusions [2].

One challenge in molecularly targeted therapy is the "moving target" of the changing proteome of cancer cells. Whether they stem from further acquisition of mutations, genetic instability, microenvironmental factors, epigenetic changes, or immunoediting, changes in cell surface protein expression of recurrent tumors may lead to resistance against targeted therapies [3-6]. In this

\footnotetext{
* Correspondence: rgorlick@montefiore.org

'Division of Pediatric Hematology/Oncology, Children's Hospital at

Montefiore, Albert Einstein College of Medicine, 3415 Bainbridge Ave,

Rosenthal Pavilion, Room 300, Bronx, NY 10467, USA

${ }^{5}$ Department of Pediatrics and Molecular Pharmacology, Albert Einstein

College of Medicine, Bronx, NY, USA

Full list of author information is available at the end of the article
}

study, we compared matched primary tumor samples to recurrent tumors to elucidate the stability of GD2 expression.

\section{Materials and methods \\ Patient samples}

An osteosarcoma tissue microarray (TMA) was constructed at Seattle Children's Hospital, Seattle, WA with prospective approval from the local Institutional Review Board. The TMA was constructed to include tumor tissue obtained at the time of initial diagnosis (from either the primary or metastatic site), at the time of definitive surgery, and at the time of disease recurrence (either local or metastatic). In total 119 cores from 57 separate specimens (of which 49 specimens were deemed useable following the construction and staining process) representing 14 different patients were included in construction of the TMA, as shown in Additional file 1: Figure S1. Each core was included as a separate data point in the analysis.

The TMAs were constructed using $2 \mathrm{~mm}$ cores obtained from the original formalin-fixed paraffin-embedded tissue block for each individual specimen. Each specimen was sampled in at least duplicate. Four micron thick unstained sections were then cut from the TMA block for subsequent immunohistochemical studies. 


\section{Immunohistochemistry}

The tissue microarray slides were stained for GD2 expression using $100 \mathrm{ug} / \mathrm{ml}$ of $14 . \mathrm{G} 2 \mathrm{a}$ antibody as previously described [2]. 14.G2a antibody was a generous gift from Dr. Karen Muszynski at the National Cancer Institute. Scoring was performed using a Nikon Inverted Microscope ECLIPSE TE200 (Nikon Instruments Inc, Tokyo, Japan) attached to a CCD (Diagnostic Instruments, Sterling Heights, Michigan) by three scientists with experience scoring tissue microarrays.

Tissue samples were scored as negative (-) if there was no staining seen. A melanoma section known to express GD2 was used as a positive control and reference for positive staining. Samples were scored as positive $(+++)$ if $67 \%$ to $100 \%$ of the section demonstrated the same intensity and distribution of staining. Slides were considered intermediate $(++)$ if $34 \%$ to $66 \%$ of cells stained positive, and were scored as sporadic $(+)$ when only $1 \%$ to $33 \%$ of cells stained positive. Tissue sections were graded by 3 independent observers blinded to the patient information. Clinical data for correlative analyses were obtained from Seattle Children's Hospital only after all grading had been completed.

\section{Statistical analysis}

Immunohistochemical assays for GD2 were assessed by three independent observers, and the level of variability between individuals was assessed using a two-factor ANOVA without replacement. When there was disagreement between the 3 observers, the median value was selected as the final tissue grading. Duplicate sample cores were assessed for agreement, and in cases of discordance, the higher value was selected as the final grading. A Mann-Whitney U-test was used to determine differences in GD2 expression levels in the primary biopsy specimens compared with the recurrent disease specimens. A $P$ value of $<0.05$ was considered to be statistically significant.

\section{Results}

\section{Demographics}

Forty-nine samples from 14 osteosarcoma patients remained for examination, taken at primary biopsy $(10 \%$ of samples), metastases at diagnosis (4\%), primary disease treated with neoadjuvant therapy at the time of

Table 1 GD2 expression in osteosarcoma samples assessed by immunohistochemistry

\begin{tabular}{llllll}
\hline & Unique cores & - & + & ++ & +++ \\
\hline Primary & 5 & 0 & 1 & 4 & 0 \\
Metastases at Diagnosis & 2 & 0 & 1 & 0 & 1 \\
Treated resection & 10 & 1 & 3 & 3 & 3 \\
Recurrent & 32 & 1 & 7 & 12 & 12 \\
\hline
\end{tabular}

definitive surgery (20\%) and at disease recurrence (65\%) (Table 1). Five patients with samples from initial biopsies with matched recurrent samples were available for evaluation in the current study; two of recurrent sample cores were from local recurrences (16\%), and the remaining sample cores were from distant lung metastases (84\%). One patient, with 2 samples from different regions of the tumor in the initial biopsy, did not have a biopsy from disease recurrence available for comparison.

Mean patient age was 14.2 years (range $7-19$ ) and $71 \%$ of patients were male. Tumor histology was classified as osteoblastic (64\%) or chondroblastic (36\%) and the most common primary tumor sites were the femur (64\%), tibia (21\%), humerus (7\%) and pelvis (7\%). All patients were treated with high-dose methotrexate, doxorubicin and cisplatin, with one patient receiving additional ifosfamide, and two patients receiving additional ifosfamide and etoposide. Additional patient characteristics can be seen in Additional file 2: Table S1.

\section{GD2 expression}

The level of variability between three independent observers was assessed to be non-significant using a twofactor ANOVA without replacement $(p=0.24)$, and the intraclass correlation coefficient was found to be 0.72 , suggesting a fair to good level of agreement. The tissue microarray of 49 samples stained with the monoclonal antibody 14G2A demonstrated GD2 expression in 95\% of samples. Ninety-seven percent of all recurrent disease specimens analyzed expressed GD2, however, the level of expression was not significantly different $(p=0.15)$ between initial biopsy samples compared with treated resection samples (Figure 1). Recurrent disease specimens demonstrated varied expression of GD2 amongst core biopsies from the same patient. Level of GD2 expression was not significantly different between initial primary biopsy specimens and matched recurrent disease specimens, whether the recurrence was local (Figure 2A) or distant (Figure 2B-D).

\section{Discussion}

Over the past few decades there has been limited improvement in outcomes for patients with osteosarcoma. The identification of specific molecular targets has the potential to improve patient outcomes with the use of novel treatment strategies. The current data demonstrate that the surface protein ganglioside GD2 is stably expressed in osteosarcoma [2]. This provides a rationale for assessing the efficacy of anti-GD2 antibody therapy in osteosarcoma patients with recurrent disease.

In contrast to the prior report, samples did not show increased levels of GD2 expression upon recurrence. Matched cores from recurrent samples showed varying expression of GD2, with no significant change of 


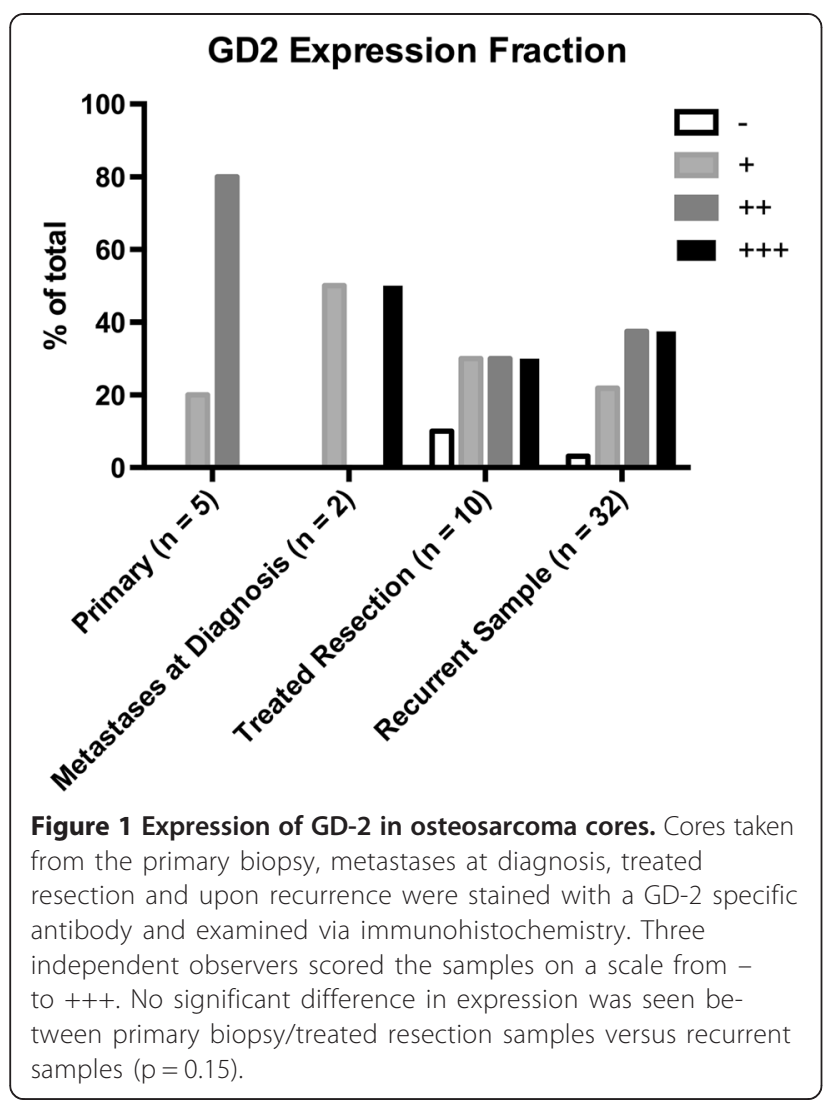

Figure 1 Expression of GD-2 in osteosarcoma cores. Cores taken from the primary biopsy, metastases at diagnosis, treated resection and upon recurrence were stained with a GD-2 specific to +++. No significant difference in expression was seen between primary biopsy/treated resection samples versus recurren expression compared to cores from the initial biopsy. The variability in expression in the cores taken at recurrence may be due to the intratumoral heterogeneity, or variability in the percentage of tumor versus stroma included in the cores, as the location of the core relative to the tumor architecture may have been reflected in variation in the local tumor microenvironment. Future studies could utilize multicolor IHC in order to identify possible intratumoral factors that influence GD2 expression. It is possible that GD2 expressing cells represent a subset of osteosarcoma cells or areas of highly proliferative cells $[7,8]$. In breast cancer, a number of groups have suggested that GD2 may be a marker of cancer stem cells, making them an especially attractive therapeutic target $[9,10]$. In such cases, examining markers for proliferation, such as Ki-67, or cancer stem cell markers, such as CD44, may elucidate potential intratumoral effects.

As therapies targeting GD2, such as the therapeutic antibody $\mathrm{CH} 14.18$, begin entering clinical testing in patients with osteosarcoma, monitoring potential loss of GD2 expression on osteosarcoma cells may aid in predicting possible development of treatment resistance. Interestingly, in patients with neuroblastoma, GD2 expression was maintained even after treatment with the anti-GD2 antibody 3 F8 [11].

The current study is limited by overall sample numbers, and it is possible that our analyses lacked the power to delineate differences in GD2 expression. However, coupled with our previous studies, it is clear that GD2 is expressed in both primary and recurrent osteosarcoma [2].

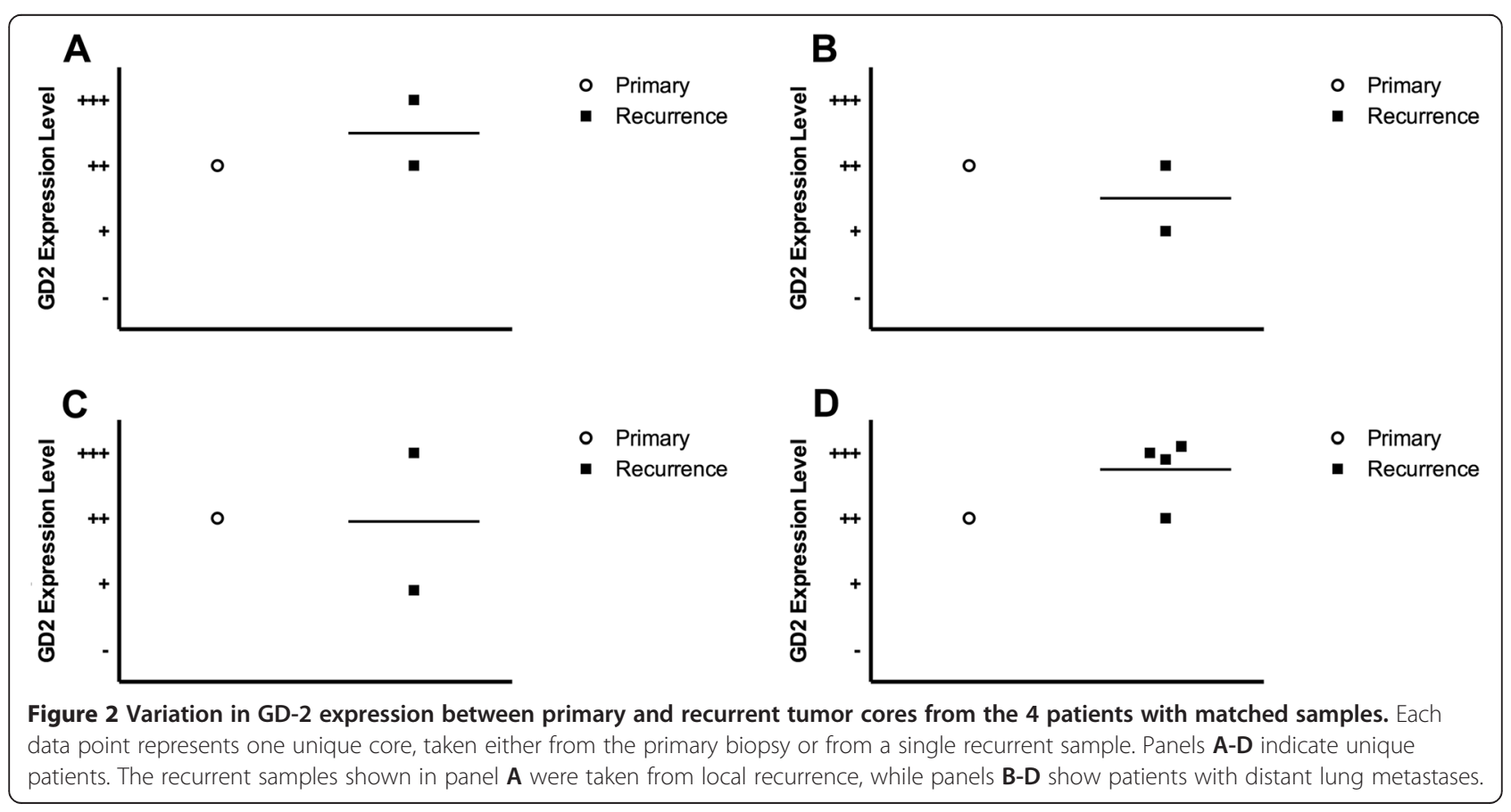


Given the near universal expression of GD2 in both primary and recurrent osteosarcoma samples, it is unlikely that GD2 expression is a prognostic biomarker, but the relatively limited number of samples examined makes this difficult to assess. However, the expression of GD2 on nearly all primary and recurrent osteosarcoma specimens suggests it is an attractive target for antibodymediated therapy. Clinical trials are needed to assess the efficacy of anti-GD2 antibody therapy in patients with osteosarcoma.

\section{Additional files}

Additional file 1: Figure S1. Workflow of the tumor biopsy sectioning, scoring, and analysis.

Additional file 2: Table S1. Clinical characteristics of the patient cohort.

\section{Abbreviation}

TMA: Tissue microarray.

\section{Competing interests}

The authors declare that they have no competing interests.

\section{Authors' contributions}

VIP performed the statistical analysis, interpreted the data and drafted the manuscript. MR conceived of the study, and participated in its design and coordination and helped to draft the manuscript. SP performed the staining and scoring of the tumor microarray. DG participated in the design and coordination of the study. JG participated in the design and coordination of the study. ERR interpreted the data and helped to draft the manuscript. DSH participated in the design and coordination of the study and helped draft the manuscript. RG conceived of the study, participated in its design and coordination and helped to draft the manuscript. All authors read and approved the final manuscript.

\section{Acknowledgement}

This research was supported by the Foster Foundation, John M. Gilbertson Foundation, the Seattle Children's Hospital Foundation, Swim Across America, and the Paul Calabresi Career Development Award for Clinical Oncology (M.R.) No. K12 CA-132783-04 from the National Cancer Institute. We would like to thank Amy Park and Tingting Ren, who assisted in the scoring of the TMA slides. We would also like to thank the National Cancer Institute for generously donating the 14.GD2a antibody.

\section{Author details}

'Division of Pediatric Hematology/Oncology, Children's Hospital at Montefiore, Albert Einstein College of Medicine, 3415 Bainbridge Ave, Rosenthal Pavilion, Room 300, Bronx, NY 10467, USA. '2Department of Orthopaedic Surgery, Montefiore Medical Center and the Children's Hospital at Montefiore, Albert Einstein College of Medicine, Bronx, NY, USA. ${ }^{3}$ Department of Pathology, Seattle Children's Hospital and University of Washington, Seattle, WA, USA. ${ }^{4}$ Division of Pediatric Hematology/Oncology, Seattle Children's Hospital, Fred Hutchinson Cancer Research Center, and University of Washington, Seattle, WA, USA. ${ }^{5}$ Department of Pediatrics and Molecular Pharmacology, Albert Einstein College of Medicine, Bronx, NY, USA.

Received: 13 November 2014 Accepted: 19 December 2014 Published online: 24 January 2015

\section{References}

1. $Y u$ AL, Gilman AL, Ozkaynak MF, London WB, Kreissman SG, Chen HX, et al. Anti-GD2 antibody with GM-CSF, interleukin-2, and isotretinoin for neuroblastoma. N Engl J Med. 2010;363:1324-34.
2. Roth M, Linkowski M, Tarim J, Piperdi S, Sowers R, Geller D, et al. Ganglioside GD2 as a therapeutic target for antibody-mediated therapy in patients with osteosarcoma. Cancer. 2014;120:548-54.

3. Chen X, Bahrami A, Pappo A, Easton J, Dalton J, Hedlund E, et al. Recurrent somatic structural variations contribute to tumorigenesis in pediatric osteosarcoma. Cell Rep. 2014;7:104-12.

4. Barsoum IB, Smallwood CA, Siemens DR, Graham CH. A mechanism of hypoxia-mediated escape from adaptive immunity in cancer cells. Cancer Res. 2014;74:665-74.

5. Anugraham M, Jacob F, Nixdorf S, Everest-Dass AV, Heinzelmann-Schwarz V, Packer NH. Specific glycosylation of membrane proteins in epithelial ovarian cancer cell lines: glycan structures reflect gene expression and DNA methylation status. Mol Cell Proteomics. 2014;13(9):2213-32.

6. Dunn GP, Bruce AT, Ikeda H, Old LJ, Schreiber RD. Cancer immunoediting: from immunosurveillance to tumor escape. Nat Immunol. 2002;3:991-8.

7. Iliopoulos D, Ernst C, Steplewski Z, Jambrosic JA, Rodeck U, Herlyn M, et al. Inhibition of metastases of a human melanoma xenograft by monoclonal antibody to the GD2/GD3 gangliosides. J Natl Cancer Inst. 1989;81:440-4.

8. Yoshida S, Fukumoto S, Kawaguchi H, Sato S, Ueda R, Furukawa K. Ganglioside $G(D 2)$ in small cell lung cancer cell lines: enhancement of cell proliferation and mediation of apoptosis. Cancer Res. 2001;61:4244-52.

9. Battula VL, Shi Y, Evans KW, Wang RY, Spaeth EL, Jacamo RO, et al. Ganglioside GD2 identifies breast cancer stem cells and promotes tumorigenesis. J Clin Invest. 2012;122:2066-78.

10. Liang YJ, Ding Y, Levery SB, Lobaton M, Handa K, Hakomori SI. Differential expression profiles of glycosphingolipids in human breast cancer stem cells vs. cancer non-stem cells. Proc Natl Acad Sci U S A. 2013;110:4968-73.

11. Kramer K, Gerald WL, Kushner BH, Larson SM, Hameed M, Cheung NK. Disialoganglioside G(D2) loss following monoclonal antibody therapy is rare in neuroblastoma. Clin Cancer Res. 1998:4:2135-9.

\section{Submit your next manuscript to BioMed Central and take full advantage of:}

- Convenient online submission

- Thorough peer review

- No space constraints or color figure charges

- Immediate publication on acceptance

- Inclusion in PubMed, CAS, Scopus and Google Scholar

- Research which is freely available for redistribution 